\title{
Risques de santé associés aux produits de poulet cru, pané et congelé : perspective d'un service de santé local
}

\author{
Julia Pinheiro Carvalho1, Mike Benusic², Raluca Codat ${ }^{3}$, Roxana Gruescu ${ }^{3}$ Liz Haydu \\ Lawrence C Loh ${ }^{2,3 *}$
}

\section{Résumé}

Depuis 2017, le poulet cru, y compris les produits congelés de poulet pané cru, a été impliqué dans 18 éclosions de salmonelle non typhoïdale au Canada. En avril 2019, l'Agence canadienne d'inspection des aliments a imposé des exigences sur l'industrie visant à réduire les risques de salmonellose dans les produits congelés de poulet cru avant leur distribution sur le marché de détail. Le présent commentaire examine les principaux problèmes identifiés par un service de santé local au cours d'une enquête sur deux cas de salmonellose survenus dans le contexte d'une récente éclosion pancanadienne liée à des produits congelés de poulet cru. Les pratiques de manipulation et de préparation des consommateurs, l'apparence des produits et les questions d'étiquetage étaient des facteurs essentiels dans le développement de la maladie. De ce point de vue de première ligne, les nouvelles exigences de l'Agence canadienne $d$ 'inspection des aliments sont analysées selon leur potentiel à réduire les risques de salmonellose dans ces produits de poulet, tout en cernant les mesures supplémentaires qui pourraient être mises en œuvre pour réduire davantage le risque d'éclosions associées à ces produits.
Cette oeuvre est mise à la disposition selon les termes de la licence internationale Creative Commons Attribution 4.0

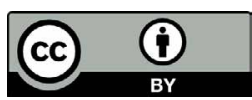

Affiliations

${ }^{1}$ Université de Sherbrooke, Sherbrooke, QC

2 Dalla Lana School of Public Health, Université de Toronto, Toronto, ON

${ }^{3}$ Santé publique de Peel, Mississauga, ON

\section{*Correspondance :}

lawrence.loh@peelregion.ca

Citation proposée : Pinheiro Carvalho J, Benusic MA, Codat R, Gruescu R, Haydu L, Loh LC. Risques de santé associés aux produits de poulet cru, pané et congelé : perspective d'un service de santé locale. Relevé des maladies transmissibles au Canada 2020;46(11/12):454-60. https://doi.org/10.14745/ccdr.v46i1112a07f

Mots-clés : salmonelle, poulet cru, sécurité alimentaire, exigences

\section{Introduction}

L'infection à la salmonelle non typhoïdale demeure l'une des principales causes de maladies d'origine alimentaire au Canada, avec une moyenne annuelle de 6881 cas signalés de 2008 à 2017, ce qui représente un taux moyen de 20 cas pour 100000 personnes par année (1). En 2018, I'incidence nationale des infections à la salmonelle a été de 19 cas pour 100000 personnes, soit une diminution importante par rapport à 2017, tandis que l'Ontario a enregistré une augmentation significative, passant de 12 à 18 cas pour 100000 personnes au cours de la même période. Salmonella enteritidis est le sérotype le plus souvent déclaré chez les Canadiennes et les Canadiennes, dans $43 \%$ des cas, suivi de Salmonella heidelberg (9\%) et de Salmonella typhimurium (6 \%) (2).

La gravité des infections à la salmonelle (aussi appelées salmonellose) peut varier, et bien que la plupart des cas se résolvent sans traitement, d'autres peuvent entraîner une grave déshydratation, une bactériémie et la mort, en particulier chez les populations vulnérables comme les jeunes enfants, les personnes âgées, les femmes enceintes et les personnes immunodéprimées (3). Le fardeau économique de la salmonellose est évalué à près de 6,43 millions de dollars annuellement en coûts de soins de santé et de 21,13 millions de dollars annuellement en pertes de productivité (4).

De nombreux produits alimentaires commerciaux contaminés par la salmonelle ont été impliqués dans des éclosions, y compris la viande, les œufs, les produits laitiers, les fruits et les légumes (3). Ces derniers temps, les produits congelés de poulet cru, tels que les languettes de poulet et les pépites de poulet, présentent un intérêt particulier car ils sont liés à des éclosions de salmonellose aux États-Unis (5), en Australie (6) et au Canada depuis les années 1990 (7). Depuis, des études ont mis en cause ces produits comme principaux facteurs de risque de salmonellose (7-10) et les données de FoodNet, un système de surveillance sentinelle dirigé par l'Agence de santé publique du Canada (l'Agence), indiquent qu'en 2018, les salmonelles ont été trouvées dans $27 \%$ des échantillons de poulet cru congelé sur ses sites (2). 
En mai 2017, le séquençage du génome entier a été introduit pour l'analyse de tous les isolats clinique de Salmonella au Canada, offrant une meilleure résolution de la parenté génétique entre les isolats de Salmonella et facilitant l'identification des foyers et éclosions de sérotype courant de Salmonelle comme l'enteridis (11). La mise en œuvre du séquençage du génome entier a permis de lier 18 éclosions de salmonellose à travers le pays ont été liées à la consommation de produits du poulet entre mai 2017 et mai 2019, ce qui a donné lieu à 584 cas confirmés en laboratoire, 97 hospitalisations, ainsi qu'au retrait de 14 produits de ce type du marché, dont 13 ont été volontairement retirés du marché par les fabricants à la suite de rappels alimentaires effectués par l'Agence canadienne d'inspection des aliments (12). De ce nombre, 12 éclosions et 285 cas ont été directement associés à l'exposition à des produits congelés de poulet pané cru (11). La région de Peel, en Ontario, a connu des tendances semblables en 2016 et 2017 , les rapports annuels d'au moins 30 enfants âgés de neuf ans ou moins développant la salmonellose après avoir consommé ces produits; un nombre qui souffre probablement d'un certain degré de sous-déclaration (13).

En réponse à ces éclosions, l'Agence canadienne d'inspection des aliments a annoncé en juillet 2018 que de nouvelles exigences seront imposées sur l'industrie en vue de mettre en œuvre des mesures de fabrication ou de transformation réduisant les concentrations de salmonelle à des niveaux non détectables dans ces produits avant leur distribution (14). Celles-ci sont entrées en vigueur le $1^{\mathrm{er}}$ avril 2019 . Le présent commentaire offre une perspective locale en matière de santé publique sur les risques associés à la consommation de produits de poulet cru, pané et congelé en décrivant les facteurs contributifs dans deux cas de salmonellose examinés par la Santé publique de Peel et en évaluant comment ces facteurs pourraient être pris en compte dans les nouvelles exigences de l'Agence canadienne d'inspection des aliments.

\section{Enquête sur une éclosion menée par un service de santé local}

Au cours de l'été 2018, les laboratoires locaux de la Santé publique de Peel ont signalé deux cas différents de salmonellose identifiés par des tests de selles, à la suite de la cascade habituelle de notification en Ontario. Chaque cas a été confié à un inspecteur de la santé publique chargé de mener des entrevues et de recueillir les échantillons nécessaires pour aider à déterminer la source de l'infection. Le premier cas était une fille de trois ans et le deuxième, un garçon de onze ans. Chaque cas présenté séparément en cas d'urgence avec fièvre et diarrhée se développant sur une période de cinq jours et chacun a fourni des échantillons cliniques pour la culture bactérienne; bien que les deux aient été renvoyés par la suite, le deuxième cas a été admis à l'hôpital pour une thérapie antibiotique intraveineuse (voir le tableau 1 pour une comparaison des cas).

\section{Tableau 1 : Comparaison des cas confirmés examinés par les agents de santé publique de Peel dans le contexte d'une éclosion de salmonellose à l'été 2018}

\begin{tabular}{|l|c|c|}
\hline \multicolumn{1}{|c|}{$\begin{array}{c}\text { Caractéristiques } \\
\text { étudiées }\end{array}$} & Cas no 1 & Cas no 2 \\
\hline Sexe & Féminin & Masculin \\
\hline Âge & Nons & Oui \\
\hline $\begin{array}{l}\text { Hospitalisation } \\
\text { requise }\end{array}$ & Cuisson au four \\
\hline $\begin{array}{l}\text { Méthode de } \\
\text { préparation du } \\
\text { produit de poulet } \\
\text { congelé impliqué }\end{array}$ & Frit dans la poêle ${ }^{a}$ \\
\hline
\end{tabular}

Dans le cas no 1, un journal alimentaire et une enquête ont révélé la consommation d'une marque nationale de pépites de poulet cru congelées et d'une autre marque de languettes de poulet précuites pendant la période d'incubation de la maladie. Les deux produits alimentaires avaient été préparés par la mère du cas conformément aux instructions figurant sur l'emballage (voir l'encadré 1 pour les instructions de cuisson des pépites de poulet). Aucune autre personne dans le ménage n'a consommé la nourriture ou n'est tombée malade. Les échantillons du repas de pépites de poulet cru, pané et congelé provenant de la maison du cas, prélevés par les inspecteurs de la santé publique et soumis au Laboratoire de santé publique de l'Ontario à des fins de dépistage, étaient positifs pour Salmonella enteritidis; les tests effectués sur les échantillons du repas de languettes de poulet précuit étaient négatifs. Le séquençage du génome entier a révélé que les isolats de l'échantillon de selles de l'enfant correspondaient à ceux des pépites de poulet pané crues congelées.

Dans le deuxième cas, un journal alimentaire et une enquête ont révélé que le mâle âgé de 11 ans consommait la même marque de pépites de poulet cru congelé que le premier cas, pendant la même période $d^{\prime}$ 'incubation. Ils n'ont pas été préparés selon les instructions de l'emballage, car ils ont été frits à la poêle avant consommation (voir l'encadré 1). Aucune autre personne dans le ménage n'a consommé la nourriture ou n'est tombée malade. Les analyses d'échantillons provenant du repas de pépites de poulet cru congelé étaient positives pour Salmonella enteritidis. Sur le séquençage du génome entier, les isolats de l'échantillon de pépites de poulet cru congelé correspondaient à ceux trouvés dans le cas no 1. Des échantillons de la même marque de croquettes de poulet pané consommé par les cas confirmés en Alberta et en Colombie-Britannique ont fourni des séquençages génomiques de Salmonella enteridis, résultant à un rappel des aliments impliqués par le fabricant (15). 
Encadré 1 : Mode de préparation dans l'emballage intérieur des pépites de poulet cru et congelé impliquées dans l'éclosion ${ }^{\text {a }}$

Ne pas mettre au micro-ondes.

Volaille crue.

Doit être entièrement cuite.

Four conventionnel :

1. Préchauffer le four à $425^{\circ} \mathrm{F}\left(220^{\circ} \mathrm{C}\right)$.

2. Mettre le poulet pané sur une plaque à biscuits antiadhésive ou légèrement graissée.

3. Cuire sur la grille au centre du four selon le temps indiqué ci-dessous :

Pépites : Cuire 8 minutes, puis retourner et poursuivre la cuisson pour 5-7 minutes

Le temps de cuisson peut varier selon l'appareil; à titre indicatif seulement. Bien cuire jusqu'à une température interne de $165^{\circ} \mathrm{F}\left(74^{\circ} \mathrm{C}\right)$.

Instructions de manipulation : $S^{\prime}$ assurer que les produits de viande et de volaille crues sont manipulés et cuits avec prudence. Garder ce produit congelé jusqu'au moment de la préparation. Garder les aliments cuits/prêts à manger à l'écart des aliments crus. Réfrigérer immédiatement les restes. Bien nettoyer les surfaces de préparation et les ustensiles, et se laver les mains après tout contact avec de la viande et de la volaille crues.

Des instructions identiques en français sont présentes à côté des instructions en anglais

\section{Discussion}

\section{Principaux problèmes identifiés au niveau local de la santé publique}

Par l'examen de ces deux cas et d'autres enquêtes antérieures, notre service de santé a cerné quatre problèmes clés susceptibles d'avoir contribué à la transmission de la salmonelle par l'intermédiaire de produits de poulet cru, pané et congelé. Premièrement, comme l'illustre le cas no 1 , il y a un risque de contamination par la salmonelle même si le produit est préparé selon les directives de l'emballage. Cela aurait pu être causé par une violation des pratiques de salubrité des aliments, comme ne pas se laver les mains avant et après manipulation des produits de poulet cru et l'absence d'assainissement des surfaces, des plats et des ustensiles après préparation et consommation des aliments.

Deuxièmement, l'apparence du produit cause un risque. Ces produits ont été préfrits par le fabricant pour donner une couleur brune à l'extérieur pané. Cela peut amener les consommateurs à croire que l'aliment est cuit $(16,17)$ et à le chauffer par la suite en utilisant d'autres méthodes de préparation, telles que le four à micro-ondes ou la poêle à frire. Ces méthodes peuvent décongeler ou chauffer inégalement le produit, sans permettre au poulet cru de cuire entièrement à la température interne recommandée de $74{ }^{\circ} \mathrm{C}(10)$. L'apparence de produits préfrits peut être particulièrement trompeuse pour les jeunes enfants, et au moins une enquête antérieure sur la salmonellose menée par notre service de santé local impliquait un enfant qui a croqué dans un produit de poulet congelé.

Troisièmement, l'inspection par les enquêteurs de santé publique a révélé que les étiquettes d'avertissement des produits concernés étaient petites et difficiles à lire. Quatrièmement, le code de lot imprimé sur la doublure en plastique interne était tamponné sur les instructions de cuisson, ce qui masquait à la fois le code de lot et les instructions. Ces détails touchant I'information fournie aux consommateurs sont particulièrement pertinents à la lumière des observations antérieures du service de santé, selon lesquelles les consommateurs déclarent avoir jeté la boîte et entreposé les pépites dans le congélateur uniquement dans le revêtement en plastique. Si les instructions de préparation ne sont imprimées que sur la boîte, elles ne sont plus accessibles au consommateur lorsque la boîte est jetée. Les consommateurs ont signalé lors d'enquêtes antérieures sur les éclosions avoir jeté la boîte parce qu'elle est encombrante et prend trop de place dans le congélateur.

Certains ou tous ces quatre facteurs ont peut-être contribué au cas no 2 , où l'enquête a révélé que les pépites avaient été préparées incorrectement à la poêle à frire. Bien qu'une raison précise d'ignorer les instructions n'ait pas été déterminée au moment de l'enquête, on peut supposer que des instructions plus claires, qui ne sont pas dissimulées et sont imprimées directement sur l'emballage intérieur, pourraient accroître la probabilité que le produit soit correctement préparé.

\section{Nouvelles mesures de contrôle}

À compter du 1'r avril 2019, l'Agence canadienne d'inspection des aliments a mis en œuvre de nouvelles exigences pour les fabricants afin de contrôler le risque de salmonellose dans les produits de poulet cru, transformé et congelé (13). Les options pour les mesures de contrôle consistent soit à inclure un processus de cuisson validé, la mise en œuvre d'un programme d'essai, une combinaison des deux, soit à mettre en place un programme de mise en attente et d'essai pour les produits finis (18). Ces exigences visent spécifiquement les produits de poulet cru, pané et préfrit non intacts destinés à la vente au détail, car ils sont plus à risque de contamination par transformation et sous-cuisson en raison de leur apparence (18). Notamment, ces exigences ne s'appliquent pas aux produits destinés à la vente aux transformateurs de services alimentaires ni aux produits de poulet pané, farci et préfrit. Bien que les exigences visent à s'attaquer à la cause première de ces éclosions, c'est-à-dire l'exposition à la salmonelle dans les produits de poulet cru, pané et congelé non farcis destinés à la 
vente au détail, elles laissent encore un certain risque résiduel de transmission par d'autres produits et par l'intermédiaire de I'industrie des services alimentaires. Le risque résiduel demeure également, car l'apparence prête à manger des produits de poulet farci préfrits pourrait continuer de donner lieu à une préparation ou à une manipulation de produits non sécuritaire.

D'autres problèmes importants qui contribuent à l'examen de notre service de santé sont liés à l'étiquetage et à l'emballage des produits et ne relèvent pas des nouvelles exigences de l'Agence canadienne d'inspection des aliments. Les mesures d'étiquetage obligatoires mises en place en 2004 exigeaient que les fabricants mettent des descripteurs tels que « non cuit " près du nom du produit et que les instructions de cuisson apparaissent sur l'emballage extérieur (17). De plus, l'Agence canadienne d'inspection des aliments, Santé Canada et l'Agence ont travaillé avec l'industrie en 2015 à l'élaboration de stratégies d'étiquetage volontaires, accompagnées d'instructions pour assurer la cohérence des messages, de mises en garde explicites contre la cuisson au micro-ondes des produits et de directives pour la cuisson sur l'emballage intérieur (17). Cependant, notre enquête a révélé que la taille de police utilisée pour l'étiquette " non cuite " était très petite et, dans le cas des pépites de poulet non cuit contaminées, elle était aussi masquée.

\section{Campagnes de sensibilisation du public}

Même si les nouvelles exigences sont un élément essentiel d'une stratégie de salubrité des aliments visant à contrer le risque d'infection par la salmonellose à partir de produits de poulet pané insuffisamment cuits, la sensibilisation des consommateurs est tout aussi essentielle. Les mesures visant à y remédier sont déjà bien en cours au niveau fédéral, où l'Agence canadienne d'inspection des aliments, Santé Canada et l'Agence ont mis sur pied d'importantes campagnes de communication pour atteindre les Canadiens par l'intermédiaire de publicités et de publications sur les médias sociaux, de la sensibilisation à d'autres médias et partenaires, et d'avis de santé publique généraux et spécifiques aux éclosions (17). De plus, en septembre 2018, les éclosions ont incité le Conseil des médecins hygiénistes en chef du Canada à publier une déclaration (voir l'encadré 2) pour informer les consommateurs de l'importance de suivre des pratiques sécuritaires de manipulation et de préparation des aliments lorsqu'ils consomment des produits congelés de poulet cru (19).

Les services de santé locaux ont également fait des efforts pour sensibiliser davantage les consommateurs à la question au moyen de la communication publique. Par exemple, en 2018, la santé publique de Peel a mené une campagne d'éducation locale dans la région de Peel pour sensibiliser les gens aux éclosions et aux divers risques liés à la consommation de produits de poulet pané cru congelé insuffisamment cuits. Cette campagne comprenait des publicités ciblées sur Google, des éditoriaux dans trois journaux locaux et des renseignements sur les réseaux sociaux et Internet sur les pratiques salubres de préparation des aliments (voir la figure 1). Depuis, le service de
Encadré 2 : Extrait de la déclaration du 13 septembre 2018 : Conseil des médecins hygiénistes en chef préoccupés par le risque de salmonellose à partir de produits congelés de poulet cru (19)

La plupart des produits de poulet pané congelés disponibles à la vente dans les épiceries au Canada contiennent du poulet cru qui peut causer la salmonellose et, par conséquent, présenter un risque accru pour la santé des Canadiens qui les manipulent, les préparent ou les consomment... Les Canadiens doivent savoir que même si ces produits peuvent sembler cuits, ils ne le sont pas.

Nous sommes très heureux que le gouvernement du Canada collabore avec l'industrie de la fabrication d'aliments et les détaillants d'aliments pour réduire la salmonelle dans les produits congelés de poulet pané cru produits le 1er avril 2019 ou après cette date à des quantités inférieures aux quantités détectables...

Cependant, jusqu'au 1er avril 2019, et probablement jusqu'à un an après cette date, les produits congelés de poulet cru contenant de la salmonelle continueront d'être sur le marché et dans les congélateurs à travers le pays. C'est pourquoi, collectivement, nous insistons sur l'importance de manipuler et de préparer avec prudence les produits congelés à base de poulet cru.

Faites toujours cuire vos produits de poulet cru congelés à une température interne d'au moins $74^{\circ} \mathrm{C}\left(165^{\circ} \mathrm{F}\right)$ selon les instructions de l'emballage en utilisant un thermomètre numérique pour aliments afin de vous assurer qu'ils sont propres à la consommation. Lavez vos mains avant et après avoir manipulé ces produits, et lavez et nettoyez les surfaces, les plats et les ustensiles utilisés pour les préparer et les servir. En suivant ces conseils, la manipulation, la cuisson ou la consommation de ces produits vous aidera à réduire les risques d'infection à la salmonelle, pour vous et votre famille. Pour obtenir plus de conseils et de renseignements sur la façon de préparer et de cuire correctement les produits de poulet cru congelé, visitez le site https://www.canada.ca/fr/sante-canada/services/risquesintoxication-alimentaire.html.

santé locale a commencé à examiner les données probantes pour déterminer les interventions efficaces visant à encourager les consommateurs à manipuler de manière salubre des aliments à domicile, dans le but de réduire le risque de maladies d'origine alimentaire découlant de pratiques de manipulation des aliments à risque dans le milieu familial.

\section{Les défis pour l'avenir}

D'importants efforts ont été accomplis pour faire face au risque de salmonellose dans les produits de poulet congelé, 
Figure 1 : Messagerie utilisée dans les journaux et la publicité numérique pour mettre en évidence les risques pour la santé associés aux produits congelés à base de poulet cru

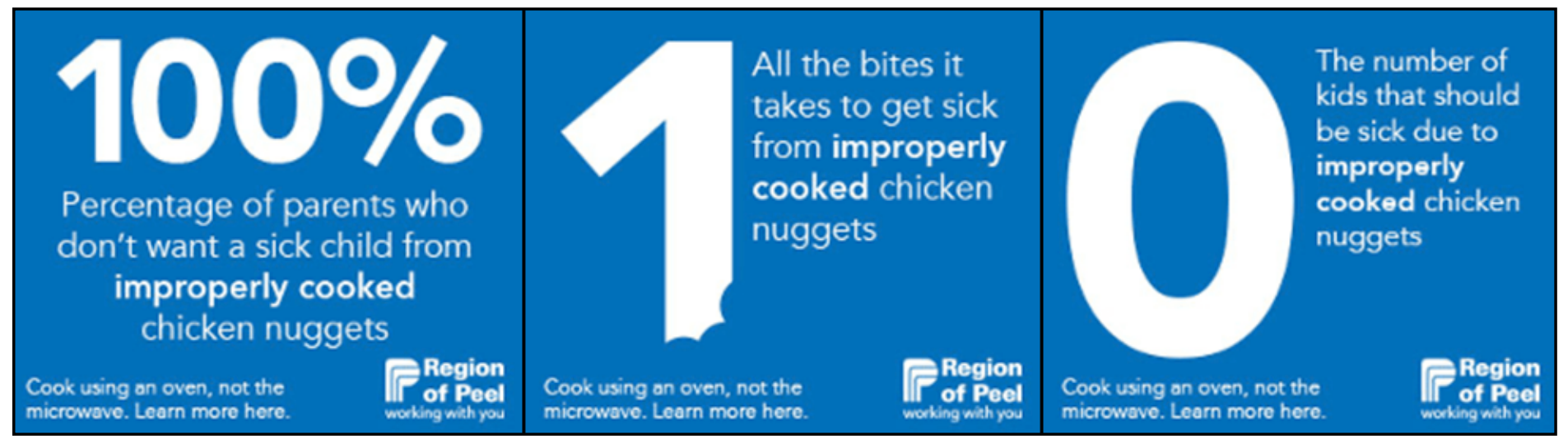

En anglais seulement

notamment des mesures préventives au niveau de l'industrie et des efforts multipartites pour sensibiliser davantage le public au problème. Cependant, comme l'a démontré l'éclosion liée aux produits congelés de poulet cru qui ont été vendus jusqu'en mai 2019 (12) et, par conséquent, après l'entrée en vigueur des nouvelles exigences de l'Agence canadienne d'inspection des aliments, les éclosions de salmonellose associées aux produits congelés de poulet cru ne sont peut-être pas encore un phénomène du passé. Il se peut fort bien que cette éclosion ait fait partie d'une période de transition. Étant donné que les produits fabriqués avant le $1^{\text {er }}$ avril 2019 pourraient encore être disponibles pendant jusqu'à deux ans sur le marché ou dans les congélateurs des consommateurs (16), l'exposition occasionnelle est toujours possible, mais l'apparition de nouveaux cas devrait diminuer au cours des prochaines années. Des cas pourraient également continuer à se présenter en association avec des produits non touchés par les nouvelles exigences, comme les produits de poulet farci.

En effet, la santé publique locale doit continuer à surveiller les maladies d'origine alimentaire, en restant vigilant sur la question de savoir si les mesures déjà prises permettront de mettre fin à ces éclosions ou si d'autres cas apparaîtront encore. Les services de santé locaux ont également un rôle à jouer dans la conduite d'enquêtes détaillées sur les cas et la détermination des nouveaux facteurs de risque, qui peuvent à leur tour être inclus dans les formulaires et documents d'enquête futurs, mis à jour régulièrement lorsque les politiques changent. Santé publique de Peel effectue également des recherches sur la meilleure façon d'informer les consommateurs sur les risques pour la salubrité des aliments à la maison, et les autorités sanitaires locales, provinciales, territoriales et fédérales doivent continuer de prendre des mesures de sensibilisation supplémentaires afin d'accroître l'adoption de mesures de salubrité des aliments par les consommateurs. Les exigences de l'industrie en matière d'étiquetage facile à lire et d'information sans obstruction sur les emballages intérieurs et extérieurs pourraient compléter les efforts de sensibilisation du public et faciliter la préparation appropriée des produits par les consommateurs. Même si une combinaison de modifications des étiquettes et de mesures de sensibilisation du public n'a apparemment pas suffi à prévenir les éclosions liées aux produits de poulet pané congelé (20), l'efficacité de ces mesures en présence des nouvelles exigences de l'industrie de l'Agence canadienne d'inspection des aliments reste à voir.

Au besoin, il y a de la place pour un travail supplémentaire visant à réduire davantage les risques. Bien que de changer l'apparence des produits de poulet pané afin qu'ils soient précuits ou ne semblent pas cuits soit un mécanisme de contrôle, d'autres opportunités de protection qui n'ont pas émergé de nos enquêtes, mais qui ont été identifiés dans d'autres éclosions canadiennes, concernent l'emballage et la commercialisation des produits. Par exemple, le fait de placer des produits non cuits à côté de produits entièrement cuits dans des congélateurs d'épicerie, l'emballage montrant le produit cuit et les produits crus qui sont commercialisés comme des repas rapides et faciles peut induire les consommateurs en erreur (7) et présenter d'autres moyens possibles de réduire les risques à l'aide d'une réglementation par les autorités sanitaires.

\section{Conclusion}

Au Canada, les éclosions de salmonellose provenant de produits de poulet pané congelé sont traitées par de nouvelles exigences au niveau de l'industrie afin de réduire le risque de contamination de ces produits par la salmonellose à des fins d'achat au détail et par des campagnes de sensibilisation du public, aux niveaux local et fédéral. Les services de santé publique sont des partenaires essentiels et continuent de s'occuper de cette question du point de vue de la surveillance locale, des enquêtes, de la collecte de données et de la collaboration avec d'autres organismes comme Santé publique Ontario et l'Agence canadienne d'inspection des aliments. Des recherches sont également menées sur la meilleure façon d'informer les consommateurs sur les risques pour la salubrité des aliments à la maison. 


\section{Déclaration des auteurs}

L. C, L., J. P. C. et M. B. ont conjointement conçu l'idée de ce manuscrit. J. P. C. a élaboré la première ébauche. Tous les auteurs ont contribué à l'élaboration et à la révision de ce manuscrit et ont approuvé le projet final pour soumission.

\section{Intérêts concurrents}

Aucun.

\section{Remerciements}

Nous remercions L. Aubin, P. Callanan, A. Chiefari et J. LeMoine pour leur contribution à la rédaction de ce manuscrit, ainsi que Mme A. Hexemer de l'Agence de la santé publique du Canada pour ses commentaires et son aide à la révision de ce manuscrit.

\section{Financement}

Aucun reçu.

\section{Références}

1. Agence de la santé publique du Canada. Nombre de cas signalés de maladies de 1924 à 2018 au Canada- maladies à déclaration obligatoire en direct. Ottawa (ON) : ASPC (modifié 2020-01-15; accédé 2020-06-29). https://maladies. canada.ca/declaration-obligatoire/graphiques?c=pl

2. Agence de la santé publique du Canada. FoodNet Canada rapport annuel 2018. Salmonella. Ottawa (ON) : ASPC; 28 mars 2019 (accédé 2020-09-08). https://www.canada.ca/ $\mathrm{fr} /$ sante-publique/services/surveillance/foodnet-canada/ publications/foodnet-canada-rapport-annuel-2018. html\#salmonella-2.1

3. Behravesh CB, Griffin PM. Salmonellosis. In: Heymann DL, editor. Control of Communicable Diseases Manual. American Public Health Association; 2015. pp. 1-2. https://ccdm. aphapublications.org/doi/book/10.2105/CCDM.2745

4. Jain S, Mukhopadhyay K, Thomassin PJ. An economic analysis of salmonella detection in fresh produce, poultry, and eggs using whole genome sequencing technology in Canada. Food Res Int. 2019;116:802-9. DOl

5. US Centres for Disease Control and Prevention. Multistate Outbreak of Drug-Resistant Salmonella Enteritidis Infections Linked to Raw, Frozen, Stuffed Chicken Entrees Produced by Barber Foods (Final Update). Washington (DC) (modifié 2015-10-16; accédé 2020-06-29). https://www.cdc.gov/ salmonella/frozen-chicken-entrees-07-15/

6. Kenny B, Hall R, Cameron S. Consumer attitudes and behaviours--key risk factors in an outbreak of Salmonella typhimurium phage type 12 infection sourced to chicken nuggets. Aust N Z J Public Health 1999;23(2):164-7. DOI PubMed
7. Hobbs JL, Warshawsky B, Maki A, Zittermann S, Murphy A, Majury A, Middleton D. Nuggets of Wisdom: Salmonella Enteritidis Outbreaks and the Case for New Rules on Uncooked Frozen Processed Chicken. J Food Prot 2017;80(4):703-9. DOI PubMed

8. Currie A, MacDougall L, Aramini J, Gaulin C, Ahmed R, Isaacs $S$. Frozen chicken nuggets and strips and eggs are leading risk factors for Salmonella Heidelberg infections in Canada. Epidemiol Infect 2005;133(5):809-16. DOI PubMed

9. MacDougall L, Fyfe M, Mclntyre L, Paccagnella A, Cordner $\mathrm{K}$, Kerr A, Aramini J. Frozen chicken nuggets and strips--a newly identified risk factor for Salmonella Heidelberg infection in British Columbia, Canada. J Food Prot 2004;67(6):1111-5. DOI PubMed

10. Middleton $D$, Savage $R$, Tighe MK, Vrbova L, Walton $R$, Whitfield Y, Varga C, Lee B, Rosella L, Dhar B, Johnson C, Ahmed R, Allen VG, Crowcroft NS. Risk factors for sporadic domestically acquired Salmonella serovar Enteritidis infections: a case-control study in Ontario, Canada, 2011. Epidemiol Infect 2014;142(7):1411-21. DOI PubMed

11. Morton VK, Kearney A, Coleman S, Viswanathan M, Chau K, Orr A, Hexemer A. Outbreaks of Salmonella illness associated with frozen raw breaded chicken products in Canada, 2015-2019. Epidemiol Infect 2019;147:e254. DOI PubMed

12. Agence de la santé publique du Canada. Avis de santé publique - Éclosions de salmonellose associées au poulet cru, dont des produits de poulet cru, pané et congelé. Ottawa (ON) : ASPC (modifié 2019-05-25; accédé 2020-06-29). https://www.canada.ca/fr/sante-publique/ services/avis-sante-publique/2018/eclosions-salmonellos e-associees-volaille-dont-produits-pouletcrus-panes-congeles.html

13. Peel Public Health. Preparing Frozen Store-Bought Chicken Nuggets. Region of Peel (ON): Peel Public Health (modifié 2018-11-05; accédé 2020-09-14). https://www.peelregion. $\mathrm{ca} /$ health/frozenchicken/index.htm

14. Agence canadienne d'inspection des aliments. Avis à l'industrie - Nouvelles exigences pour réduire la quantité de Salmonella à un seuil inférieur aux quantités décelables dans les produits de poulet crus panés et congelés. Ottawa $(\mathrm{ON})$ : ACIA (modifié 2019-04-01; accédé 2020-09-08).

https://www.inspection.gc.ca/salubrite-alimentaire-pour-|industrie/directives-archivees-sur-lesaliments/produits-de-viande-et-de-volaille/ changements-au-programme/2018-07-12/ fra/1520884138067/1520884138707

15. Agence de la santé publique du Canada. Modalités canadiennes d'intervention lors de toxi-infection d'origine alimentaire (MITIOA): Guide d'intervention en cas d'éclosion multijuridictionnelle de maladie entérique. Ottawa (ON) : ASPC (modifié 2018-02-21; accédé 2020-09-08). https:// www.canada.ca/en/public-health/services/publications/ health-risks-safety/canadas-foodborne-illness-outbre ak-response-protocol-fiorp-guide-multi-jurisdictio nal-enteric-outbreak-response.html 
16. Santé Canada. Poulet cru pané et surgelé. Ottawa (ON) : SC (modifié 2019-02-06; accédé 2020-06-29). https://www. canada.ca/fr/sante-canada/services/salubrite-viande s-volailles-poissons-et-fruits-mer/poulet-pane-surgele.html

17. Agence canadienne d'inspection des aliments. Questions et réponses: Nouvelles mesures pour réduire la salmonelle dans les produits de poulet. Ottawa (ON) : ACIA (modifié 2019-04-01; accédé 2020-06-29). https://www. inspection.gc.ca/controles-preventifs/produits-de-viande/ salmonella-dans-les-produits-de-poulet-crus-panes-/faq/ fra/1554140834819/1554140994648

18. Agence canadienne d'inspection des aliments. Options de contrôle de Salmonella dans les produits de poulet crus panés et congelés. Ottawa (ON): Gouvernement du Canada (modifié 2019-04-01; accédé 2020-06-29). https:// www.inspection.gc.ca/controles-preventifs/produits-deviande/salmonella-dans-les-produits-de-poulet-crus-panes-/ fra/1531254524193/1531254524999
19. Agence de la santé publique du Canada. Le risque de salmonellose associé aux produits de poulet crus, panés et congelés préoccupe les membres du Conseil des médecins hygiénistes en chef. Ottawa (ON) : ASPC (modifié 201809-14; accédé 2020-06-29). https://support.extyles.com/ support/solutions/articles/1000178077-upon-attempting-t o-run-activate-and-normalize-on-a-document-word-crashe s-or-the-document-doesn-t-act

20. Smith KE, Medus C, Meyer SD, Boxrud DJ, Leano F, Hedberg CW, Elfering K, Braymen C, Bender JB, Danila RN. Outbreaks of salmonellosis in Minnesota (1998 through 2006) associated with frozen, microwaveable, breaded, stuffed chicken products. J Food Prot 2008;71(10):2153-60. DOI PubMed 